Article published in: Schulte Nordholt A., Smith P.J. (Eds.) Jeux de mots enjeux littéraires, de François Rabelais à Richard Millet. Essais en hommage à Sjef Houppermans. Leiden - Boston: Brill. p. 45-67.

\title{
Variations sur le nom de Rabelais
}

Paul J. Smith (Université de Leyde)

Le premier des innombrables jeux de mots de Rabelais porte sur son propre nom. On le rencontre sur la page de titre de Pantagruel, son premier ouvrage de fiction (Figure 1), publié à Lyon, probablement en 1532. François Rabelais y présente son nom sous forme d'anagramme : «Composez nouvellement par maistre Alcofrybas Nasier ${ }^{1}$. Depuis, Rabelais lui-même ainsi que ses lecteurs - admirateurs et adversaires - ne cessent de jouer sur ce pseudonyme. Ce sont ces déformations onomastiques, à travers les siècles, du temps de Rabelais jusqu'aujourd'hui, que je me propose d'étudier dans le présent article.

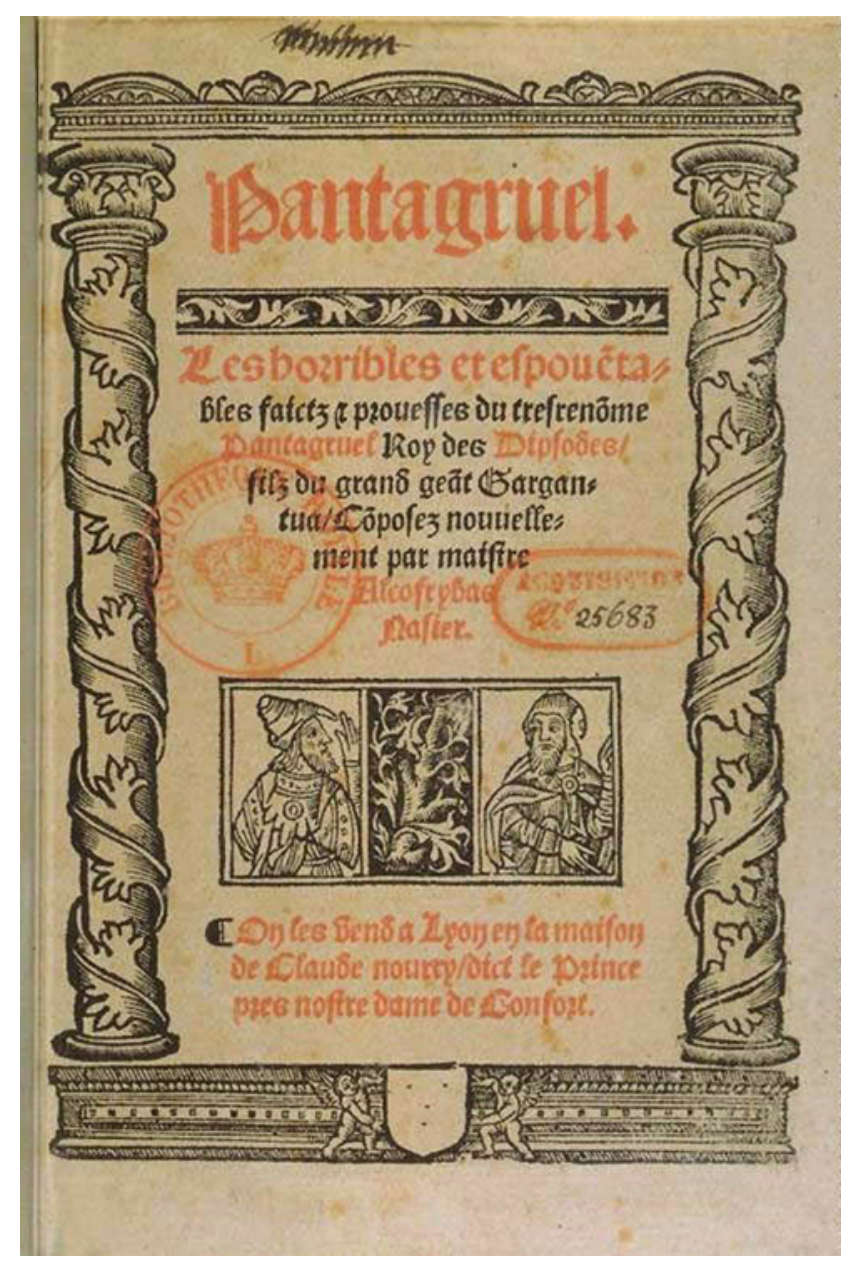

\footnotetext{
${ }^{1}$ Mes références sont à Rabelais, Euvres complètes, Mireille Huchon (éd.), Paris, Gallimard, Bibliothèque de la Pléiade, 1994.
} 


\section{Figure 1. Rabelais, Pantagruel ( $1^{\mathrm{e}}$ édition, sans date), page de titre. Bibliothèque nationale de France, Rés $Y^{2} 2146$.}

\section{Alcofrybas Nasier}

Les raisons pour écrire sous pseudonyme, et sous ce pseudonyme précis, restent peu claires. S'agit-il d'un anonymat de prudence, qui serait de mise pour un auteur débutant ? Cette hypothèse est peu convaincante, l'anagramme étant trop transparente pour cacher le nom de l'auteur, qui publie, également en 1532, coup sur coup, trois livres savants en latin, parmi lesquels une édition de Galien, dont les épîtres dédicatoires exhibent fièrement son nom et son titre fraîchement acquis : "Franciscus Rabelaesus Medicus ». Cependant, on a relevé récemment la relative opacité du pseudonyme. Comme le dit Romain Menini : «Qui associait les chroniques gigantales à l'éditeur d'Hippocrate et de Galien ? Personne, si ce n'est les amis, les protecteurs, le monde du livre lyonnais et quelques happy few, du Poitou et d'ailleurs $»^{2}$. Quoi qu'il en soit, ce lectorat semble trop restreint pour croire à un pseudonyme de prudence. En effet, on va le voir, certaines réactions de lecteurs prouvent que dès 1533, Rabelais a été reconnu comme l'auteur de Pantagruel. Il convient d'assumer que Rabelais adopte une stratégie auctoriale concertée, qui consiste à publier d'un seul coup tout ce qu'il avait écrit jusque-là, dans le but de conquérir par attaque-surprise son public visé. Le poète Joachim du Bellay procédera de la même façon, lorsqu'il publie, en 1558, cinq recueils à la fois, dont un en latin (Poemata), les quatre autres en français (Les Regrets, Les Antiquitez de Rome, Les jeux rustiques et Songe). Dans cette stratégie, Rabelais cherche, moyennant l'anagramme, à différencier entre les deux côtés de son œuvre de « docte gabeur », l'un sérieusement humaniste et savant et l'autre grotesquement comique - dichotomie que Mireille Huchon, dans son Rabelais (2011), a rapprochée de l'effet Dr. Jekyll and Mr. Hyde ${ }^{3}$. Ce faisant, Rabelais semble se souvenir de deux de ses maîtres à penser, Erasme et Thomas More, qui publient, parmi leurs œuvres savantes, des ouvrages moins sérieux. Ainsi Erasme fait paraître son Éloge de la Folie anonymement, mais sous l'égide de Stultitia, la Folie, et si More publie son Utopie sous son propre nom, il a hâte de reléguer la voix narrative à un narrateur dont le nom bipartite Raphaël Hythlodaeus présente une coincidentia oppositorum, combinant le sérieux et le ludique : Raphaël est l'archange qui guérit Tobie de cécité, l'accompagne en ses voyages et est, partant, patron des voyageurs, tandis que « Hythlodaeus » signifie en grec « expert en mensonges ». L'ambiguïté joue aussi dans l'anagramme rabelaisienne, comme le montre François Rigolot:

Par son profil arabisant, « Alcofrybas » se rattache à la lignée des Avicenne, Albenmasser et Ali-ben-el Abbas, tous Abstracteurs de Quintessence. [...] « Alcofrybas », l'Abstracteur, a la tête dans les nuages, mais « Nasier », le renifleur, a

\footnotetext{
${ }^{2}$ Romain Menini, «Rabelais, homme d'atelier(s)», Isabelle Garnier e.a. (éds.), Paroles dégelées. Propos de l'Atelier XVIe siècle. Paris, Classiques Garnier, 2016, pp. 451-470 (p. 454). Olivier Pédeflous, " Rabelais et un lexicographe des années 1530, Wolfgang Hunger », Garnier e.a. (éds.), Paroles dégelées, o.c., pp. 547-561 (p. 555).

${ }^{3}$ Mireille Huchon, Rabelais, Paris, Gallimard, 2011, p. 136. Ou comme le dit Menini, « Rabelais, homme d'atelier(s) », art. cit., p. 461 : « Docteur Rabelais contre Mister Alcofribas ».
} 
bien les pieds sur terre ; si le premier préfère l'huile de la science, le second sait humer le bon vin ${ }^{4}$.

Interprétation à laquelle les lecteurs du XVI siècle auraient sans doute souscrit. Ainsi, Johann Fischart, le grand traducteur et imitateur de Rabelais en Allemagne, énumère, pour souligner la soi-disante respectabilité du nom d' "Alcofrybas », pas moins de 25 savants dont le nom commence par le préfixe « $\mathrm{Al}-{ }^{5}$ - énumération comique, parce que Fischart ajoute des noms comme « Alexander » (le Grand) et « Albertus » (Magnus) (dont « Al- » n' est pas un préfixe), il déforme des noms existants et bien connus («Alristotel », « Alnoldus »), et il en invente d'autres pour le besoin de la cause. Et la connotation nasale de « Nasier » ne passe pas non plus inaperçue, ce dont témoigne le poème héroï-comique Naseïde, publié par Bérenger de la Tour d'Albenas en 1556 (reprise en 1558) ${ }^{6}$. Cette Naseïde, « dediée au grand Roy Alcrofribas Nazier », commence ainsi : «Pour vous louer si la plume je prens, / Roy des grans nez, roy des nes les plus grans / De Naseri [...] $\gg^{7}$. Nous sommes ici en présence d'une thématique grivoise (où le nez peut être le signe du buveur ou faire allusion au membre viril) qui trouve son origine dans les plaisanteries qu'a faites Ovide (Naso) sur son propre nom.

Il est probable que le nom de « Nasier » comporte une réminiscence autobiographique qui renvoie aux contacts du jeune Rabelais avec son mentor et ami Jean Thenaud, savant franciscain au service de la reine-mère Louise de Savoie ${ }^{8}$. A l'instigation de Louise, Thenaud écrit en 1517-1518 son Triumphe des vertus, un volumineux miroir de prince encyclopédique, destiné à l'éducation des deux enfants de Louise : le jeune François ${ }^{\text {er }}$ et sa sœur Marguerite. Ce texte allégorique, resté manuscrit, comporte nombre d'anagrammes, selon le goût de la cour. Parmi les nombreux personnages allégoriques que le narrateur autodiégétique rencontre, se trouve un jeune homme, nommé Nazaire, qui initie le narrateur à la littérature comique (François Villon et la Farce de Maistre Pathelin), dont Rabelais, on le sait, savoure la lecture. Ce Nazaire est cher au narrateur (l'alter ego de Thenaud) : « Nazaire, bon enfant d'effect et de nom, mon bon ami, lequel jadiz j'avoye congneu plus feal que ne fut Damis à Appoloneus ». Mon hypothèse est que ce « bon amy » est le jeune Rabelais, qui, graecus précoce et talentueux, aide Thenaud dans ses lectures des œuvres comiques de l'auteur grec

\footnotetext{
${ }^{4}$ François Rigolot, Poétique et onomastique. L'exemple de la Renaissance, Genève, Droz, 1977, p. 84. 5 « Meister Alguckzaunuß, Alcofribas, Albategnus, Albahasses, Alfraganus, Alveroes, Alzarcheles, Alfonsus, Alperragus, Alexander, Alquisinus, Doctor Albram, Alristotel, Alvenatzre, Alchelaus, Algips, Alrabs, Alyaces, Alnaces, Albertus, Alquinus, Alchindus, Albentragel, Alponensis, Alnoldus unnd Alnarholdus » (Johann Fischart, Aller Practick Grossmutter [...], s.l.n.n., 1593, Vorred, sans indication de page).

${ }^{6}$ Ce poème est en fait une traduction de la Nasea (1539) d'Annibal Caro. Les allusions à Rabelais ne se trouvent pas dans le texte italien, mais sont le fait du traducteur. Voir Franco Giacone, « Rabelais et Annibal Caro : traditions, filiations et traductions littéraires ", Revue d'Histoire littéraire de la France 99 (1999), pp. 963-973.

${ }^{7}$ Cité et traduit par Marcel De Grève, L'interprétation de Rabelais au XVIe siècle, Genève, Droz, 1961, p. 112.

${ }^{8}$ Le présent paragraphe résume l'essentiel de Paul J. Smith et Titia J. Schuurs-Janssen, «'Plus feal que ne fut Damis à Appoloneus'. Rabelais et Jean Thénaud avant 1517 : quelques hypothèses », dans Marie-Luce Demonet et Stéphan Geonget (éds.), Les grands jours de Rabelais en Poitou [...], Genève, Droz, 2006, pp. 183-194.
} 
Lucien de Samosate et dans la rédaction du Triumphe ainsi que d'autres textes. En effet, « Nazaire » est phoniquement lié à « Nasier», et traduits en latin, ces deux noms donneraient « Nazarius ». En utilisant le nom de « Nasier », Rabelais actualise un souvenir de sa jeunesse passée en Poitou, un souvenir aussi de son amitié avec Thenaud. Il le fait aussi ailleurs dans son œuvre. Ainsi, Rabelais semble faire écho à la tournure « plus feal que ne fut Damis à Appoloneus », en faisant dire à Panurge, qui, à la fin du Tiers Livre, s'adresse à Pantagruel : « Je vous seray un Achates, un Damis, et compaignon en tout le voyage. Je vous ay long temps congnue amateur de peregrinité et desyrant tous jours veoir, et tous jours apprendre » (pp. 494-495). Rabelais semble penser ici à Thenaud, qui parle lui-même de sa «peregrination », et se nomme " pouvre pellerin et desireux explorateur ». Et, on va le voir, l'œuvre de Rabelais comporte d'autres allusions à Thenaud, tout comme l'œuvre de Thenaud, à son tour, montre parfois de remarquables ressemblances avec celle de Rabelais, similitudes qui s'expliquent par l'aide rédactionnelle que le jeune Rabelais avait donnée à son ami' .

La formule anagrammatique qui figure sur la page de titre de la première édition de Pantagruel, «Composez nouvellement par maistre Alcofrybas Nasier », reste inchangée dans les autres éditions de Pantagruel parues en 1532 et 1533 . Cependant, les pages de titre des éditions successives de Pantagruel et des autres livres de Rabelais nous apprennent que cette formule est sujette à des changements importants. Dans l'édition de François Juste (Lyon, 1533), on ajoute à la formule «Composez nouvellement par maistre Alcofrybas Nasier » la précision suivante : " Augment[é] et Corrig[é] fraischement, par maistre Jehan Lunel docteur en theologie ». Ce Jehan Lunel est inconnu. Est-ce une allusion obscure au théologien italien Lunel (avec qui Rabelais n'avait pas eu de contact, pour autant qu'on sache), ou s'agit-il d'une simple allusion à la nature lunatique que possèdent, selon la veine satirique des érasmiens, nombre de théologiens?

Cet ajout énigmatique reste un cas isolé, restreint à cette édition de 1533. Par contre, les autres éditions de Pantagruel, parues du vivant de Rabelais, montrent d'autres remaniements onomastiques qui, édition après édition, se standardisent, sans doute avec le consentement de Rabelais. Ainsi, le nom de « Nasier » disparaît, et on ajoute les qualificatifs « feu » et « abstracteur de quinte essence ». Voici donc l'évolution :

- « composez par M. Alcofribas abstracteur de quinte essence » (éditions de 1534 et $1535)$;

- « composez par feu M. Alcofribas abstracteur de quinte essence » (éditions entre 1537 et 1542 ;

- $\quad$ une édition de 1537 remplace « composez » par « enchronicquez »).

Cette évolution, par le mot « feu », désactualise le moment de la création, suggérée dans les premières éditions par les qualificatifs «nouvellement » et « fraischement » : elle confère à Alcofrybas une ancienneté vénérable, qui s'accorde bien avec les savoirs secrets en alchimie que l'Abstracteur de quinte essence est censé posséder.

\footnotetext{
${ }^{9}$ Voir Paul J. Smith, « Jean Thenaud and François Rabelais: Some Hypotheses on the Early Reception of Erasmus in French Vernacular Literature », dans Karl A.E. Enenkel, The Reception of Erasmus in the Early Modern Period, Leyde-Boston, Brill, 2013, pp. 211-236.
} 
Ces développements autour du nom d'Alcofrybas s'arrêtent brusquement dans l'édition de Claude La Ville (Valence, 1547) : le pseudonyme disparaît pour laisser la place au nom de l'auteur: « composés par M. Franç. Rabelais, Docteur en Medecine ». Cette appellation, qui est, à première vue, d'une parfaite transparence, est néanmoins mystifiée par le qualificatif qui suit, et qui laisse le lecteur moderne perplexe : « et Calloier des Isles Hieres ». Mireille Huchon suggère que, possiblement, Rabelais fait ici une autre allusion à Thenaud («Clin d'œil à un ami cher? ») : en effet, Thenaud, « dans Le voyage et itineraire de oultre mer, composé vers 1520, parle longuement des « caloyers qui sont moynes grecz », après une première étape aux îles d'Hyères » (p. 299). ${ }^{10}$

Les changements onomastiques que l'on constate sur les pages de titre des éditions de Pantagruel sont en rapport avec ce qu'on voit dans les éditions des autres livres de Rabelais. Ainsi, les pages de titre de Gargantua (première édition sans lieu ni date, mais probablement imprimée en 1535) répètent jusqu'en 1547 : «jadis composé par L'abstracteur de quinte essence » ou « jadis composee par M. Alcofribas abstracteur de quinte essence ». La Pantagrueline Pronostication (sans lieu ni date, mais datant probablement de 1533) ajoute un nouvel élément dans les éditions parues du vivant de Rabelais : " Maistre Alcofribas Architriclin dudict Pantagruel » - le qualificatif « architriclin » (maître d'hôtel) souligne l'ambiguïté d'Alcofrybas, qui n'est pas le simple avatar anagrammatique de l'auteur, mais qui, au service du personnage Pantagruel, appartient aussi à la diégèse du livre. Et, conformément au Pantagruel de l'édition de Valence de 1547, le pseudonyme «Alcofrybas Nasier » disparaît pour de bon des éditions du Tiers Livre (douze éditions entre 1546 et 1553) et du Quart Livre (quatre éditions partielles en 1549, neuf éditions en 1552 et 1553).

\section{Alcofrybas entre auteur et personnage}

Toutes ces modifications onomastiques reflètent les changements qui s'effectuent, d'un livre à l'autre, dans les rapports entre auteur, présentateur des prologues, narrateur et personnage. Dans les premières éditions de Pantagruel, du moins jusqu'à celle de 1542, le nom d'Alcofrybas s'applique aux quatre fonctions narratives à la fois, créant ainsi une ambiguïté narrative. Ainsi, la voix du « Prologue de l'Auteur » n'est pas celle de l'auteur « réel », id est Rabelais, mais celle du narrateur homodiégétique et du personnage fictionnel, comme le souligne ce Prologue :

C'est des horribles faictz et prouesses de Pantagruel, lequel j'ay servy à gaiges dés ce que je fuz hors de page, jusques à present, que par son congié je m'en suis venu visiter mon pais de vache, et sçavoir si en vie estoyt parent mien aulcun. (p. 215)

En effet, Alcofrybas retourne dans la narration comme personnage, qui dialogue avec Panurge, qui l'appelle « mon amy » (chapitre XVII), et avec Pantagruel, qui s'adresse à lui, lorsqu'il sort de sa bouche: « « Dont viens tu, Alcofrybas ? » Je luy responds. « De vostre gorge, monsieur.» » (p. 333). Cet Alcofrybas est identique à l'Alcofrybas sur la page de titre.

\footnotetext{
${ }^{10}$ Par ailleurs, Huchon, Rabelais, o.c., p. 299, propose aussi d'autres hypothèses qui lient Rabelais aux îles d'Hyères.
} 
Cependant, cette page de titre est « défictionnalisée » dès que le nom d' « Alcofrybas » est remplacé par « Franç. Rabelais » dans l'édition de Valence de 1547.

L'ambiguïté narrative tourne en aporie dans Gargantua : la voix du Prologue y est celle de Rabelais (un Rabelais défendant comiquement son livre), et se distingue de la voix du narrateur extradiégétique anonyme ${ }^{11}$ tel qu'il se présente comme le déchiffreur/traducteur philologue des « Fanfreluches antidotées » (pp. 10-11). Il n'est pas présent comme personnage dans la narration : la seule mention du nom d' " Alcofrybas » semble concerner quelqu'un d'autre : « [l'anneau] fut faict par le capitaine Chappuys et Alcofribas son bon facteur [commis]» $\gg$ p. 27) ${ }^{12}$.

Dans les Tiers et Quart Livres la situation narrative se désambigüise : les mentions de « François Rabelais » sur les pages de titre correspondent à la voix de l'auteur dans les prologues et les autres paratextes, alors que, dans la partie fictionnelle de ces livres, Alcofrybas redevient narrateur autodiégétique et personnage. Cependant, dans ces deux derniers livres, Rabelais brise encore une fois la fictionnalité narrative : ses personnages mentionnent « Rabelais » lorsqu' ils évoquent deux souvenirs particulièrement émotifs, l'un joyeux, l'autre triste. Joyeux, lorsqu'ils se rappellent la représentation théâtrale de la farce de « la femme mute », jouée par les étudiants - « nos antiques amys » - de Montpellier, parmi lesquels est mentionné François Rabelais. Le personnage Epistémon, qui était là comme spectateur, se rappelle: « Je ne riz oncques tant, que je feis à ce Patelinage » (p. 460). L'autre souvenir, triste, est également évoqué par Epistémon. Il concerne les «prodiges tant divers et horrificques » qui ont précédé la mort de Guillaume du Bellay, le protecteur bien aimé de Rabelais. Epistémon se rappelle comment les personnes présentes à l'agonie de Du Bellay - il en mentionne seize, parmi lesquelles « Rabelays » - « et tant d'aultres amis, domesticques, et serviteurs du deffunct tous effrayez se reguardoient les uns les aultres en silence sans mot dire de bouche » ( pp. 602-603).

\section{Seraphin Calobarsy et Bacabery l'aisné}

À deux reprises Rabelais expérimente avec d'autres anagrammes de son nom. Le premier cas se rencontre dans Gargantua : si le nom d' «Alcofrybas Nasier » est quasi absent dans ce livre, on y trouve, du moins dans les premières éditions, «Seraphin Calobarsy », anagramme de « Phrancoys Rabelais ». Ce nom est utilisé à un moment important dans le livre : le début du chapitre XXIII, intitulé « Comment Gargantua feut institué par Ponocrates en telle discipline, qu'il ne perdoit heure du jour ». Ce chapitre décrit comment Ponocrates, le nouveau précepteur humaniste, fait table rase de toute l'éducation sophistique que le jeune géant a reçue jusque-là. Pour ce faire, il « supplia un sçavant medicin de celluy temps, nomm[é] Seraphin Calobarsy ». Ce médecin applique un médicament efficace, l'ellébore, qui guérit la folie :

Lequel purgea canoniquement avec Elebore de Anticyre, et par ce medicament luy nettoya toute l'alteration et perverse habitude du cerveau. Par ce moyen aussi

\footnotetext{
${ }^{11}$ Il n'est pas clair s'il est identique à l'Alcofrybas de la page de titre.

${ }^{12}$ Allusion plaisante à Claude Chappuy, un des amis de Rabelais. Sur « Alcofribas », Mireille Huchon note : «C'est le seul cas dans l'œuvre où ce nom est cité, et il apparaît comme un personnage différent du narrateur de Gargantua. » (Rabelais, o.c., p. 1087).
} 
Ponocrates luy feist oublier tout ce qu'il avoit apris soubz ses antiques precepteurs. (p. $64)$.

Cette anagramme, utilisée à cet endroit précis du livre, est loin d'être innocente. Tout se passe, en effet, comme si c'était Rabelais lui-même qui intervenait dans sa fiction de façon décisive. C'est que, à partir de ce moment-là, la tonalité du récit se transforme radicalement : de satire comique (visant la mauvaise éducation sophistique et « sorbonagre ») elle change en éloge sérieux (prônant la bonne éducation humaniste). Dès ce moment aussi, l'humour change de cap : le jeune géant cesse d'être un personnage risible. Il devient un exemple à suivre, incarnant la sagesse humaniste, qui ne prête guère plus à rire. Aussi, le rire sera-t-il généré par certains autres personnages, et plus particulièrement par frère Jean dans Gargantua, ainsi que frère Jean et Panurge, et non pas Pantagruel - tel père tel fils -, dans les Tiers et Quart Livres. Cependant, s'il arrive à Rabelais d'être sérieux, il ne l'est jamais à part entière. Selon Lazare Sainéan, dans son étude ancienne mais séminale, « Calobarsy » serait « une transposition de $\kappa \alpha \lambda$ ó $\beta \dot{\rho} \sigma \alpha$, belle outre à vin (Lucien), digne pendant des « buveurs tresillustres » $»^{13}$.

La question est de savoir pourquoi, dans les éditions de Gargantua à partir de 1535, Rabelais a remplacé « Seraphin Calobarsy » par «maistre Theodore » [Don de Dieu]. Ce ne serait sans doute pas pour cause de prudence ou de crainte de Rabelais envers les autorités universitaires ou ecclésiastiques. Selon Michael Screech, la raison réside dans le fait qu'une anagramme semblable, "Seraphino Calbarsy », a été utilisée sur la page de titre d'une pronostication sérieuse, dont Rabelais ne fut pas l'auteur ${ }^{14}$ : Rabelais voulait « se distancer de ces nouvelles pronostications, peu conformes à ses propres conceptions du rôle de l'astronomie scientifique $»^{15}$.

La seconde anagramme se trouve dans le Quart Livre, à la fin d'un catalogue de morts extraordinaires. Sont nommées les grandes autorités : Verrius, Pline, Valère Maxime, Fulgose. Cette énumération se termine par « Bacabery l'aisné », que l'on a interprété comme l'anagramme de « Rabelais cy en ba[s] », c'est-à-dire « du soussigné Rabelais ». Nous suivons ici l'interprétation d'André Tournon : « Il est fort possible qu'il [Rabelais] se moque du lecteur à l'affût d'indices et de mystères ; l'hypothèse, à ne jamais négliger, ne dispensera pas de jouer le jeu, et d'essayer de comprendre cette attestation, en songeant à la fonction assignée à l'épisode ». Cette fonction est comparable à celle de l'anagramme Seraphin Calobarsy : il s'agit d'introduire le sérieux, qui est, le cas échéant, le thème du trépas ${ }^{16}$.

\section{Rabie laesus}

Le nom de Rabelais prête facilement aux déformations linguistiques faites par ses lecteurs contemporains, tant amis qu'ennemis. ${ }^{17}$ En cela ils suivent la pratique de Rabelais lui-même.

\footnotetext{
${ }^{13}$ Lazare Sainéan, La langue de Rabelais, Genève, Slatkine Reprints, 1976 (Paris, 1922-1923), volume II, p. 416.

${ }^{14}$ Signalons cependant ici l'hypothèse récente de Mireille Huchon, pour qui Rabelais, « selon toute vraisemblance », serait l'auteur de cette pronostication. Voir Huchon, Rabelais, o.c., pp. 168-172.

${ }^{15}$ Voir cette pronostication, éditée par M.-A. Screech, dans Etudes Rabelaisiennes 15 (1980), pp. 179209 (p. 194).

${ }^{16}$ André Tournon, « Nargues, Zargues et le concept de trépas », Réforme, Humanisme, Renaissance 64 (2007), pp. 111-123 (p. 112).

${ }^{17}$ On passe sous silence les nombreuses déformations non-intentionnelles du nom de Rabelais, telles que nous avons trouvées, par exemple, dans les catalogues de vente aux enchères au XVIIe siècle : Rabbellays, Rablais, Rabelaesi, Rabbelais, Rablaeis. Voir Paul J. Smith, « Rabelais aux Pays-Bas :
} 
Rabelais, par moquerie bienveillante, déforme les noms de certaines personnes admirées : ainsi il transforme Thomas More en Thaumaste, l'occultiste Corneille Agrippa en Herr Trippa, le médecin Guillaume Rondelet en Rondibilis, et le juriste André Tiraqueau en Trinquamelle. La moquerie de Rabelais devient acerbe lorsqu'il s'agit de ses détracteurs. Ainsi, il fustige Guillaume Postel (qui avait qualifié le « Pantagruellus » et l' " Abbatia Thelemeton » (l'abbaye de Thélème) de créations impies et luthériennes), Jean Calvin (qui avait attaqué Rabelais dans son livre Des scandales) et Gabriel du Puy-Herbault (dont le Theotimus accuse Rabelais d'obscénité et d'impiété) en déformant leurs noms et en les mettant ensemble parmi les enfants d'Antiphysie (Anti-Nature) (sans différencier entre catholiques zélés et réformateurs genevois): « les Maniacles Pistoletz, les Demoniacles Calvins imposteurs de Geneve, les enraigez Putherbes [...], et aultres monstres difformes et contrefaicts en despit de Nature » (p. 615).

Curieusement, le premier qui critique Rabelais en jouant sur son nom, fut un ami : Nicolas Bourbon. Dans une épigramme latine In Rabellum, parue dans ses Nugae (1533), celui-ci reproche à l'auteur de distraire, par ses écrits, les écoliers de leurs études. Citons la pointe de ce poème dans la traduction française de Marcel De Grève : «Crois-moi, Rabelais, laisse dès à présent nos écoliers recouvrir leur santé morale, de crainte que les Muses, que tu persécutes de tous côtés comme un furieux, ne se jouent de toi ici-bas et ne te fassent sombrer dans la rage $»^{18}$. La traduction ne rend pas justice au jeu de mots sur « rabies » (folie, rage, et plus particulièrement la rage transmise par des morsures de chien) et « Rabelais » dans la proposition finale : "Ac ne te in rabiem inferant Rabelle ». Selon De Grève ${ }^{19}$, les vers célèbres de Rabelais, « Mieulx est de ris que de larmes escripre / Pource que rire est le propre de l'homme » (p. 3) seraient une réplique au «puritanisme naissant » de Bourbon et d'autres. Dans une édition ultérieure des Nugae, Bourbon a omis cette épigramme maladroite, mais le mal était déjà fait : depuis ce moment les lecteurs de Rabelais liaient son nom à « rabies ».

Cela vaut aussi pour un autre ami de Rabelais, Jean Visagier qui, dans ses Epigrammatum Libri II (1536), tout en critiquant et corrigeant Bourbon, étend le jeu de mots de ce dernier. ${ }^{20}$ Citons ce poème, qui lie « rabies » à « laesus » (« atteint de », « pris de ») :

\section{Ad Rabelaesum}

Qui rabie assuruit laesum, Rabelaese, tuum cor

Adjunxit vero cum tua Musa sales,

Hunc puto mentitum, rabiem tua scripta sonare

l'édition Elzevier (1663) et la présence de Rabelais dans les bibliothèques privées des Hollandais », dans id. (éd.), Editer et traduire Rabelais à travers les âges, Amsterdam-Atlanta, GA., Rodopi, 1997, pp. 141-173 (pp. 162-166).

${ }^{18}$ De Grève, L'interprétation de Rabelais, o.c.., p. 17.

${ }^{19}$ Id., p. 79.

${ }^{20}$ Je passe sous silence le nom de "Rabella" que l'on trouve dans les épigrammes "Ad Rabellam" dans les Inscriptionum libri duo (1538) de Visagier et "In Rabellam" (1553) de Nicolas Chesneau. Selon Lucien Febvre, ces épigrammes s'adressent non pas à Rabelais, mais à Étienne Dolet. Voir Lucien Febvre, Le problème de l'incroyance au XVIe siècle. La religion de Rabelais, Paris, Albin Michel, 1968 (1942), pp. 65-68. Cependant, le rapport entre Rabella et Rabelais a été fait, par un lecteur inconnu contemporain, qui, dans son exemplaire du recueil de Visagier, a écrit, à côté de "Rabella", le nom de François Rabelais (voir Febvre, p. $65)$. 
Qui dixit: rabiem, dic, Rabelaese, canis?

Zoïlus ille fuit, rabidis armatus iambis ;

Non spirant rabiem, sed tua scripta jocos. ${ }^{21}$

Ce « rabie laesus », avec la connotation de « chien enragé » ${ }^{22}$, est tout de suite utilisé par les détracteurs de Rabelais, parmi lesquels Calvin, qui écrit en 1555 : « ce [Rabelais et semblables moqueurs] sont des chiens enragez qui desgorgent leurs ordures à l'encontre de la majesté de Dieu $»^{23}$.

Le nom de Rabelais prête à d'autres déformations onomastiques, dont par ailleurs il n'est pas toujours certain qu'il visent Rabelais. Ainsi, on trouve le nom de « Rabella » dans les épigrammes «Ad Rabellam » dans les Inscriptionum libri duo (1538) de Visagier et « In Rabellam » (1553) de Nicolas Chesneau. Selon Lucien Febvre, ces épigrammes, où il est question d'un personnage parasitaire et hypocrite, s'adressent non pas à Rabelais, mais à Étienne Dolet. ${ }^{24}$ Cependant, quoi qu'en dise Febvre, le rapport entre « Rabella » et Rabelais a été fait, une seule fois au moins, par un lecteur inconnu contemporain, qui, dans son exemplaire du recueil de Visagier, a écrit, à côté de « Rabella », le nom de François Rabelais. ${ }^{25}$ (voir Febvre, p. 65). Febvre a trouvé dans les Ludi (1538) d'un certain Hubert Sussannée un poème intitulé « In Rabulam » (rabula en latin est un orateur frénétique), et deux autres : « In Rubellum » et « Ad Rubellum » (rubellus en latin signifie « tirant sur le rouge », adjectif souvent utilisé pour qualifier le vin). Ces trois épigrammes s'adressent probablement à Rabelais, mais sans malice.

Le poète Pontus de Tyard fait encore un autre calembour sur le nom de Rabelais, basé sur l'hébreu rab ou rabbi ( «maître ») et lez (« plaisanterie ») :

Is est famosus ille Rabelez, qui ab Hebraeis dictionibus [...] Rab, et [...], lez, verissime irrisorum principis nomen habuit, quod Gallicè dixeris, un maistre mocqueur. Id vero cognomen fato, ut mihi videtur, quodam illi (quamvis ex genere et familia) est inditum. ${ }^{26}$

[Celui est le célèbre Rabelez, qui a tiré son nom des mots hébraïques Rab et lez, est nommé ...]

\footnotetext{
${ }^{21}$ Jean Visagier cité et traduit par De Grève, L'interprétation de Rabelais, o.c., p. 30 : «Celui qui a prétendu, Rabelais, que ton cœur était infecté de rage, alors que ta Muse s'est contentée d'épicer la vérité - celui-là a menti en disant que tes écrits respiraient la rage. La rage, dis-nous, tu la chantes donc, Rabelais ? Non, c'est lui, ce Zoïle, qui s'est muni d'iambes enragées ; tes écrits à toi, ce n'est pas la rage, c'est les ris qu'ils respirent $»$.

${ }^{22} \mathrm{C}$ 'est ce que Visagier semble suggérer en utilisant la forme verbale « canis » (tu chantes), qui est l'homophone du substantif « canis » (chien).

${ }^{23}$ Calvin cité par De Grève, L'interprétation de Rabelais, o.c., p. 90.

${ }^{24}$ Voir Lucien Febvre, Le problème de l'incroyance au XVIe siècle. La religion de Rabelais, Paris, Albin Michel, 1968 (1942), pp. 65-68.

${ }^{25}$ Louis Thuasne, Études sur Rabelais, Paris, E. Bouillon, 1904, p. 315. Voir Febvre, Le problème de l'incroyance, o.c., p. 65.

${ }^{26}$ Pontus de Tyard, De recta nominum impositione, Lyon, Jacob Roussin, 1603, p. 27 , cité par Katia Campbell, «Rabelais and the Massorah », dans Barbara C. Bowen (éd.), Rabelais in Context, Birmingham, Alabama, Summa Publications, 1993, pp. 55-66 (p. 55). Les crochets indiquent ici l'omission des caractères hébraïques.
} 
Au siècle suivant, un admirateur de Rabelais, Antoine Le Roy, proteste contre cette étymologie, qu'il trouve mal plaisante, en proposant une autre : «Potest autem meo quoque iudicio vocari Rabelaesus, quasi Rabby laesus, hoc est Magister et Doctor offensus quorundam imperitorum ac malevolorum iniuriis [...] $»^{27}$ [Mais, selon moi, Rabelais pourrait être appelé tout aussi bien Rabby laesus, c'est-à-dire Maître et Docteur souffrant des injures de certains ignorants et malveillants]. De même, Jean Bernier, dans Le véritable Rabelais réformé (1697), souligne qu'il désapprouve ces faciles calembours : « Il y a des gens de si grand loisir, qu'il s'en est trouvé qui ont voulu joüer sur son nom par des anagrammes, des étymologies \& des allusions, qui ne font rien à nôtre sujet $»^{28}$.

\section{Epithètes, périphrases}

La réputation de Rabelais suscite l'inventivité lexicale de ses lecteurs non seulement dans le domaine de l'anagramme et du calembour, mais aussi dans celui de l'épithète et de la périphrase. Ainsi, Ronsard parle du « bon Rabelais », et Joachim Du Bellay invente l'épithète « utile-doux ». Le rapprochement avec les auteurs satiriques de l'Antiquité, surtout Lucien, est fait par de nombreux auteurs admirateurs et détracteurs : Du Bellay (« Celuy, qui faist renaistre Aristophane, et feint si bien le nez de Lucian »), Henri Estienne (" nostre siècle a faict revivre un Lucian en un François Rabelais, en matière d'escrits brocardant toute sorte de religion»), Étienne Pasquier (« Lucianus alter » et le « Lucien de notre temps, Rabelais ») ${ }^{29}$. Dans son dictionnaire d'épithètes, publié en 1571, Maurice de La Porte énumère les épithètes faites sur Rabelais tout en ajoutant un bref commentaire :

\section{Rabelais. Facetieus, mordant, utile-doux, raillard, second Epicure, gausseur ou gaudisseur, lucian françois, docte gabeur, ventre epicurien, plaisant moqueur, pantagruelite.}

François Rabelais docteur en medecine, tant par son livre qu'il a intitulé les Euvres de Pantagruel, que par sa voluptueuse vie, a merite ces epithetes : qui voudra avoir de lui plus grande connaissance, lise les epithaphes qu'en ont composé plusieurs sçavans personnages. ${ }^{30}$

Ce disant, La Porte fait entre autres allusion à Ronsard, qui a composé un célèbre épitaphe sur le « bon Rabelais, qui buvoit / Tousjours ce pendant qu'il vivoit ». Dans ce poème Ronsard reprend et amplifie l'image que Rabelais a créée de lui-même : celle d'un buveur et d'un mangeur insatiable.

\footnotetext{
${ }^{27}$ Antoine Le Roy, Floretum philosophorum [...], Paris, I. Dedin, 1649, sans pagination.

${ }^{28}$ Jean Bernier, Jugement et nouvelles observations sur les æuvres [...] de François Rabelais, ou Le véritable Rabelais réformé. Avec la carte du Chinonois, Paris, L. d'Houry, 1697, p. 2.

${ }^{29}$ Pour un survol récent de ces dénominations avec des références bibliographiques utiles, voir Nicolas Corréard, « Les « Histoires vraies » du «Lucien français : de la poétique de l'incrédulité au regard moraliste du Quart Livre », Le Verger -bouquet 1, janvier 2012 (http://cornucopia16.com/wpcontent/uploads/2014/07/Verger-1_CORREARD.pdf (dernière consultation 5-9-2016)).

${ }^{30}$ Maurice de La Porte, Les Epithetes, Paris, Gabriel Buon, 1571, p. 224.
} 
Ces connotations permettent à l'un des amis de Rabelais, Bonaventure des Périers, de lui donner une place dans l'un des textes les plus obscurs du XVI ${ }^{\mathrm{e}}$ siècle, le Cymbalum Mundi (1537). Rabelais y figure (ou semble y figurer, car l'identification n'est pas soutenue par tous les critiques) sous le personnage d'un chien, appelé « Pamphagus », à côté des personnages anagrammatiques moins opaques, comme Rhetulus (Luther(us)), Cubercus (le réformateur Bucer(us)) et Drarig (palindrome dénotant probablement le théologien Girard Roussel) ${ }^{31}$. Le nom de « Pamphagus » [Dévore-tout] tiré des Métamorphoses d'Ovide, présente de vagues ressemblances phoniques avec certains noms rabelaisiens, ayant le préfixe « Pan- ». Ce nom est encore utilisé par Joachim du Bellay, probablement pour désigner Rabelais $^{32}$.

Cependant, à la fin du siècle, ce mot semble se dissocier de la figure de Rabelais. Il devient un mot commun, ce dont témoigne l'entrée « Pamphagus » dans l'une des sources lexicales les plus importantes de l'époque, A Dictionarie of the French and English Tongues (1611; plusieurs éditions) par Randell Cotgrave, qui note : «Pamphage. Eat-all, all-ravening, all-devouring ».

\section{Variations tardives sur Alcofrybas}

Ce dernier exemple montre par ailleurs qu'après la mort de Rabelais les variations sur son nom commencent à mener leur propre vie. L'exemple le plus spectaculaire est le nom même d'Alcofrybas. Si pour Cotgrave, « Alcofrybas » signifie simplement « A greedie glutton. A great devourer », le nom, dans le contexte flamand, continue à connoter la narration facétieuse. Ainsi, dans une adaptation flamande d'une version en prose du Roman de Renart, le nom du magicien « Abrion de Trier » est remplacé par celui d' " Alcofrybas », « meester Alcofribras [sic pour le /r/] (een seer gheleert Jode in alle consten ende talen)» [maitre Alcofribas, un Juif très savant en tous les arts et langues]. Dans cette même édition flamande du Roman de Renart, on trouve une autre allusion à Rabelais : « Meester Robbicolio, den broeder van Noscorqui » [Maître Robbicolio, le frère de Noscorqui], qui fait écho, par simple mutation de /a/ en /o/, à « Rabelais » et « Nasier ». Il est à remarquer que dans les éditions ultérieures de la version flamande, le nom de « Noscorqui » subit de nombreuses variations : « Nazergus », « Nazorkwi », « Nestorqui », « Nestoroni », lesquels se distancient progressivement de « Nasier », avant de disparaître complètement dans les éditions du XVIII ${ }^{\mathrm{e}}$ siècle ${ }^{33}$.

Toutefois, au XIX ${ }^{\mathrm{e}}$ siècle, Alcofrybas réapparaît dans le roman pour la jeunesse Histoire du célèbre Pierrot écrite par le magicien Alcofribas, traduite du sogdien (1860) d'Alfred Assollant, et illustré par un célèbre illustrateur, Yan Dargent, dont les gravures ne sont pas sans faire penser à celles que Gustave Doré a faites pour illustrer Rabelais. Ce livre, dont certains personnages (Pantafilando, entre autres) présentent de vagues similitudes avec les personnages rabelaisiens, connaît un certain succès. Non seulement il sera traduit en

\footnotetext{
${ }^{31}$ Ce n'est pas le lieu ici d'argumenter cette identification. Nous renvoyons à Mireille Huchon, Rabelais, o.c., pp. 261-264.

32 Joachim Du Bellay, "Pamphagi medici", cité, traduit et commenté par De Grève, L'interprétation de Rabelais, o.c., pp. 103-104.

${ }^{33}$ Voir Is. Teirlinck, "Reinaert en Rabelais", Verslagen en Mededeelingen der Koninklijke Vlaamsche Academie voor Taal- en Letterkunde. Januari 1904, Gand, Sipper, 1904, pp. 37-49.
} 
anglais par A.G. Munro (1875), mais Alcofribas sera aussi le héros éponyme d'un courtmétrage muet, L'enchanteur Alcofribas (1903), de Georges Méliès. En outre, « Alcofribas » est le nom du «Loup Foque » dans une bande dessinée de la revue Spirou en 1975, et plus récemment, dans un livre pour enfants, intitulé Alcofribas et la méduse noire de Pierre Crookx (2010), le nom est donné à une bande de trois enfants et un chien.

Alcofrybas continue à tracasser les esprits des philologues. En témoigne l'Edition variorum, la première édition critique des Euvres de Rabelais, publiée en 1823. Si leur travail proprement philologique est sérieux, les deux éditeurs, Charles Esmangart et Eloi Johanneau, dans les notes de leur édition, font preuve d'une érudition joyeuse dans l'interprétation des deux noms « Alcofrybas » et « Nasier ». En éditant Gargantua, ils confèrent d'abord une origine espagnole à « Alcofrybas » :

il semble qu'il ait vu, dans le premier [Alcofrybas], un mot espagnol compose de algo, quelque peu, ou quelque chose, et de bribar, gueuser, mendier, briber, brifer, friper, qui bribe, brife, ou fripe quelque peu, un fripe-lippe ou fripe-sauce, qui aime la fripe, les franches lippées, un liguritor en latin; et dans le second [Nasier], un mot françois dérivé du latin nasus, nase ou nez, qui a du nez, pour sentir et flairer, au sens propre et au figuré. ${ }^{34}$

A la page suivante, cette interprétation tourne rapidement à un délire interprétatif, qui est inconsciemment (?) scabreux :

Alcofribas pourroit bien aussi, dans l'idée de l'auteur, être composé de al, article arabe de composition, en espagnol, pour el, de co, préposition également de composition, pour con ou cum, et de fribas, dans le même sens que ci-dessus ; et signifier le conlécheur (conliguritor) ; le con-fripeur, le compagnon ou le co-ami de la fripe, c'est-àdire de la friandise ; le con-bribeur, soit parcequ'il aimoit en effet les bonnes bribes, les bons morceaux, la bonne chère, et le bon vin, soit parcequ'il avoit été ou qu'il étoit peut-être encore de l'ordre mendiant des cordelier, à Fontenay, et que bribe signifioit panis mendicatus ; bribeur, mendicus ; briber, mendicare, comme le marque Nicot. ${ }^{35}$

Cependant, plus tard, en éditant le chapitre XXXII de Pantagruel (qui relate le voyage fait par Alcofrybas dans la bouche de Pantagruel), ils reviennent sur cette interprétation, à laquelle ils substituent une interprétation onomastique grecque tout aussi délirante :

Il y a déjà long-tems que nous avons reconnu que nous nous étions trompés sur l'étymologie que nous en avions donnée au commencement du premier volume, et nous allons profiter de cette occasion pour en présenter une nouvelle, qui nous a été suggérée par une lecture attentive de ce chapitre. Nous pensons qu'il a composé le

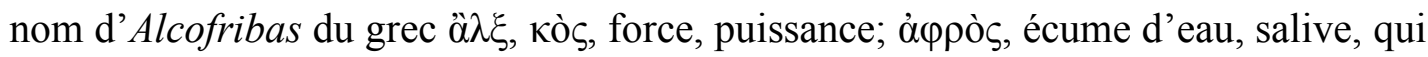

\footnotetext{
${ }^{34}$ Rabelais, Euvres. Edition variorum [...], éds. [Charles] Esmangart et Eloi Johanneau, tome Ier, Paris, Dalibon, 1823, p. 8, n. 3.

${ }^{35}$ Idem, p. 9 , n. 3.
} 
fait en composition $\alpha \dot{\varphi} \rho 1$, et $\beta \alpha \zeta$, qui va, qui va ou marche dans une forte salive. C'est ainsi qu'il a formé les mots de nephelibate, qui va dans les nues; d'alibantes, qui marchent dans le sel, et d'almyrodes, les salés. Ce nom factice convenoit très bien à un gorgias qui avoit demeuré dans la gorge de Pantagruel, au chastelain de Salmigodin, c'est-à-dire à Rabelais, qui habitoit alors à Fontenai-le-Comte, ou à Maillezais, ou à Montpellier, près les marais Salans. Tout ce chapitre [le chapitre XXXII de Pantagruel] est la confirmation de cette étymologie. ${ }^{36}$

\section{Fischart anagrammatique}

C'est sous la plume de Johann Fischart que l'on retrouve un surplus de déformations comiques du nom de Rabelais. Curieusement, cela ne se fait pas encore sur les pages de titre de Geschichtklitterung, la traduction, ou plutôt l'adaptation, en allemand de Gargantua, dont voici la page de titre de la dernière édition revue par l'auteur, celle de 1590 (Figure 2) :

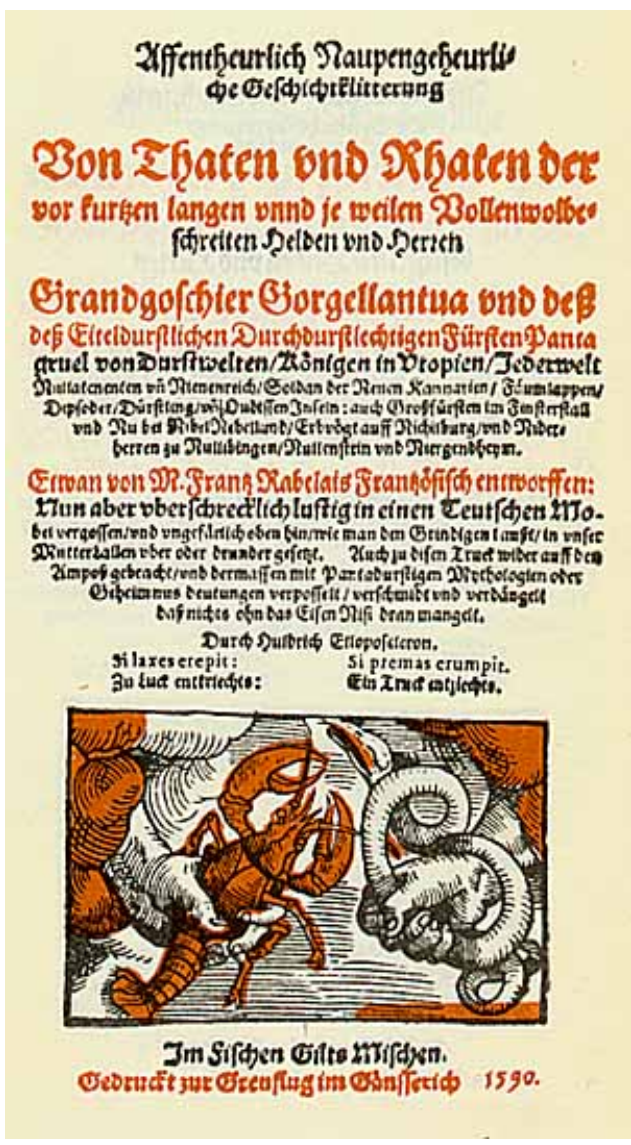

Figure 2. Johan Fischart, Geschichtklitterung, 1590, page de titre (source:

http://www.hs-augsburg.de/ harsch/germanica/Chronologie/16Jh/Fischart/fis gkti.jpg).

En transcription, qui fait justice à la mise en page :

\footnotetext{
${ }^{36}$ Idem, p. 141, n. 36.
} 


\title{
Affentheurlich Naupengeheurli- che Geschichtklitterung
}

\author{
Von Thaten und Rhaten der \\ vor kurtzen langen unnd je weilen Vollenwolbe- \\ schreiten Helden und Herren \\ Grandgoschier Gorgellantua und deß \\ deß Eiteldurstlichen Durchdurstlechtigen Fürsten Panta- \\ gruel von Durstwelten, Königen in Utopien, Jederwelt \\ Nullatenenten und Nienenreich, Soldan der Neuen Kannarien, Fäumlappen, \\ Dipsoder, Dürstling, und OudissenInseln: auch Großfürsten im Finsterstall \\ und $\mathrm{Nu}$ bel NibelNebelland, Erbvögt auff Nichilburg, und Nider- \\ herren zu Nullibingen, Nullenstein und Niergendheym. \\ Etwan von M. Frantz Rabelais Frantzösisch entworffen: \\ Nun aber überschrecklich lustig in einen Teutschen Mod- \\ el vergossen, und ungefärlich oben hin, wie man den Grindigen laußt, in unser \\ MutterLallen über oder drunder gesetzt. Auch zu disen Truck wider auff den \\ Ampoß gebracht, und dermassen mit Pantadurstigen Mythologien oder \\ Geheimnus deutungen verposselt, verschmidt und verdängelt \\ daß nichts ohn das Eisen Nisi dran mangelt. \\ Durch Huldrich Elloposcleron.
Si laxes erepit:
Si premas erumpit.
$\mathrm{Zu}$ Luck entkriechts:
Ein Truck entziechts
$[\ldots]$
Im Fischen Gilts Mischen

Je dis « curieusement », parce que les autres noms propres sur cette page de titre ont subi une vertigineuse déformation comique. Ainsi, Grandgousier est traduit par « Grandgoschier », nom qui contient « Gosch » (bouche) - ce qui, par ailleurs, s'accorde bien avec l'élément « gousier» (gorge) dans « Grandgousier ». « Grandgoschier » annonce un grand nombre d'autres déformations du nom dans Geschichtklitterung - le glossaire de l'édition moderne d'Ute Nyssen ${ }^{37}$ en mentionne douze, mais en réalité le nombre est plus élevé. De même, sur la page de titre de Geschichtklitterung, «Gargantua » devient « Gorgellantua » (avec jeu de mots sur « gorge(1)n» ou « gurgeln » (se gargariser)), et Nyssen mentionne cinq autres variantes du nom dans le reste du texte : « Garkantenvoll» (jeu sur « Kanne » (verre) et « voll » (plein)), « Gargantzsoffa » (jeu sur « saufen » (boire)), « Gurgelstrossa », « Gurgelstrozza » (référence à « Strosse » (gorge)), et « Gargelzipflin » (« Zipf » signifie « pépie ») ${ }^{38}$. Sur la page de titre, « Pantagruel» est d'abord combiné avec les formations adjectivales « Durchdurstlechtigen » (contamination de « Durst » (soif) et « durchl(a)uchtig » (sérénissime)) ; puis le nom donne lieu à la forme adjectivale «Pantadurstigen » (« durstig » signifie « assoiffé »). Mais les déformations les plus spectaculaires sur cette page de titre, Fischart les fait sur son propre nom - suivant en cela l'exemple de Rabelais/Alcofrybas, mais

\footnotetext{
${ }^{37}$ Ute Nyssen, Glossar [zu] Johann Fischart, Geschichtklitterung, Düsseldorf, Karl Rauch Verlag, 1964, p. 17.

${ }^{38}$ Pour ces interprétations, voir Nyssen, Glossar, o.c., p. 17.
} 
de manière démesurée. Fischart remotive son nom de famille en le découpant de deux manières au moins, représentées visuellement dans l'emblème sur la page de titre :

(1) « Fisch » (poisson) + Art (espèce) $:$ donc « espèce de poisson ». Cette première manière l'amène à la traduction de son nom en grec : «Elloposcleron », qui signifie littéralement « espèce de poisson ». L'emblème de la page de titre représente deux espèces de poissons : une anguille et une écrivisse ${ }^{39}$, dont la dernière a une carapace dure (ce qui correspond à un autre découpage du nom : « Fisch » + « hart » (dur)).

(2) « fisch(en) » (pêcher) + Art (manière) : donc " manière de pêcher » ou « art de pêcher ». L'emblème représente les deux manières opposées pour attraper et tenir le poisson dans la main.

Ces deux manières de pêcher (2) dépendent de deux espèces de poissons (1) : pour tenir l'anguille, on ne presse pas la prise, sinon elle s'échappe en glissant. Par contre, l'écrivisse s'échappe si l'on lâche la prise. Cette opposition antithétique est mise en un distique latin : « Si laxes erepit: Si premas erumpit», traduit en allemand : « Zu Luck entkriechts: Ein Truck entziechts ». Ce distique fonctionne comme le motto dans la structure tripartite de l'emblème dans la tradition du Liber emblematicum d'Alciat (1534), à savoir : motto, pictura (image), subscriptio. La subscriptio, le dernier élément constituant de l'emblème, se réduit ici à un énoncé en allemand "Im Fischen Gilts Mischen », qui est un acrostiche du nom de l'auteur : J.F.G.M. : « Johann Fischart genannt Mentzer ». Cet énoncé signifie : « pour bien pêcher, il convient de mélanger ». Il est non seulement applicable au jeu onomastique sur le nom de Fischart, mais aussi sur le titre même du livre Geschichtklitterung : "Klitterung » peut se traduire par « mélange ». En effet, par cet emblème onomastique, le livre de Fischart se présente, dès sa page de titre, comme un mélange, tant au niveau de sa production :

« fischen » et « mischen » indiquant la manière d'écrire de Fischart (Fisch-Art), que de sa lecture : il convient au lecteur de changer et d'adapter sa prise sur Fischart selon sa pêche interprétative. ${ }^{40}$

Dans ces virtuoses tourbillons onomastiques qui figurent sur la page de titre, le caractère inchangé du nom de François Rabelais saute aux yeux : il n'y a que la traduction de « François » en « Frantz » qui amène à une facile association de mots : «Frantz Rabelais Frantzösisch ». Mais cela ne dure pas : dès qu'on continue la lecture du livre, on s'aperçoit que le nom de Rabelais n'échappe pas non plus à la déformation joyeuse de Fischart. Dans une communication personnelle, Elsa Kammerer, qui prépare un livre sur Rabelais et Fischart ${ }^{41}$, m'a signalé trois exemples remarquables :

\footnotetext{
${ }^{39}$ Selon les ichtyologues de l'époque (Belon, Rondelet), l'écrivisse appartient à la catégorie mal définie des « poissons ».

${ }^{40}$ On a beaucoup écrit sur cette page de titre, son emblème et son interprétation poétologique. Voir, entre autres, Florence M. Weinberg, Gargantua in a Convex Mirror. Fischart's View of Rabelais, New York - Bern - Frankfurt a. M. et al., 1986, p. 15. Pour une interprétation récente de cet emblème, voir Tobias Bulang, « Hermeneutic Animals. Johann Fischart's Use of Emblems in His German Translation of Rabelais », dans Karl A.E. Enenkel et Paul J. Smith (éds.), Emblems and the Natural World 1500-1700, Leyde-Boston, Brill (à paraître en 2017).

${ }^{41}$ Elsa Kammerer, Vberschrecklich lustig. Rabelais sur le métier de Fischart (1575-1590), livre en préparation. Je remercie Elsa Kammerer de ces informations très utiles à mon argumentation.
} 
(1) l'énoncé « Rabiles res Mirabiles », qui met en rapport le nom de « Rabelais » et le vers « O res mirabilis » (o chose admirable !) de l'hymne Panis angelicus de Thomas d'Aquin ;

(2) la déformation de « rabelistisch » en « Rabelistig », qui joue sur le mot « listig » (malin, astucieux) ;

(3) le rapprochement de « Rabelais » et « rabulisch », qui est la forme adjectivale de « Rabulistik ». ${ }^{42}$

Précisons par ailleurs que ce dernier rapprochement est comiquement paradoxal, parce que Rabelais s'attaque justement à la sophistique de la Sorbonne. L'élément « Rabulisch » est intégré dans une forme adjectivale plus longue : «Brockarttrabulisch », forme complexe (on reconnaît, outre « Rabelais » et « rabulisch », « Brokat », « Brock », « Art »), qui est censée traduire l'adjectif rabelaisien « robidilardique ».

On ne s'étonne pas que le nom d'Alcofrybas ne soit guère déformé par Fischart, tout simplement parce que ce nom ne figure pas sur la page de titre de Geschichtklitterung (Fischart a utilisé une édition tardive de Gargantua, qui n'a pas le nom d'Alcofrybas sur sa page de titre). La seule mention d'Alcofrybas dans Gargantua (« et Alcofribas son bon facteur ») est traduit par « und den Biervogt Alcofribras ». Le second « $\mathrm{r} »$ dans «Alcofrybas » n'est certes pas une simple coquille ${ }^{43}$ : elle réfère probablement au verbe « brassen » (boire beaucoup), ce qui s'accorde avec « Biervogt », où l'on reconnaît « Bier » (bière) et « Vogt» (patron).

\section{Epilogue : АГАОН ТYХН, à la bonne fortune}

Le nom de Rabelais et ses prolongements anagrammatiques et épithétoires continuent à préoccuper les lecteurs de notre époque. On pense au roman The Rebel Angels (1981) de Robertson Davies qui intègre le nom de Rabelais (prononcé à l'anglaise) dans son titre et le nom d'Urquhart, le célèbre traducteur anglais de Rabelais, dans celui de l'un de ses personnages. Et dans le domaine de la critique rabelaisienne, on pense à l'intérêt nouveau suscité par la devise de Rabelais, АГА $Н \mathrm{TYXH} \mathrm{(à} \mathrm{la} \mathrm{bonne} \mathrm{fortune),} \mathrm{que} \mathrm{certains} \mathrm{de} \mathrm{ses}$ ouvrages portent sur leur page de titre. Claude La Charité et Romain Menini, dans le sillage de Pierre-Paul Plan et de Mireille Huchon ${ }^{44}$, ont démontré que cette devise appartient uniquement à Rabelais, et non pas à l'un des imprimeurs lyonnais. La question est de savoir quelle a été l'intention de Rabelais pour choisir cette devise. Menini propose d'y lire une référence à Erasme, qui dans ses Apophtegmata signale que c'est la devise de l'orateur Démosthène, qui, préférant l'eau au vin, personnifie, dans l'œuvre de Rabelais, le versant labeur dans la création littéraire. Citons le texte d'Erasme dans la traduction de Menini :

\footnotetext{
${ }^{42}$ Comme on l'a vu dans le cas de Sussannée, le rapprochement entre « Rabelais » et « Rabulus » a été fait bien avant Fischart. Il n'est pas clair si Fischart en était au courant.

${ }^{43}$ Contrairement donc à la coquille dans la version flamande du Roman de Renart, citée plus haut.

${ }^{44}$ Claude La Charité, Rabelais éditeur du Pronostic. "La voix véritable d'Hippocrate », Paris, Classiques Garnier, à paraître ; Pierre-Paul Plan, Bibliographie rabelaisienne. Les éditions de Rabelais de 1532 à 1711, Paris, Imprimerie nationale, 1904, p. 233 ; Mireille Huchon, Rabelais grammairien. De l'histoire du texte aux problèmes d'authenticité, Genève, Droz, 1981, p. 347, et son édition de Rabelais, Euvres, o.c., p. 1229, note 1.
} 
Démosthène, sur son bouclier, avait fait inscrire en lettres d'or : $\alpha \gamma \alpha \theta \dot{\eta}$ $\dot{\chi} \chi \eta$, c'est-àdire à la bonne fortune. Mais alors qu'on en venait aux prises, il jeta son bouclier surle-champ et s'enfuit. Lorsqu'on lui en fit grief, en lui remontrant le déshonneur qu'il y a à être un lâche-bouclier, il se joua du reproche en citant le petit vers bien connu : Un fuyard combattra de nouveau. Il indiquait qu'il était plus utile à la patrie de fuir que de mourir au combat. ${ }^{45}$

Démosthène qui se dérobe, c'est selon Menini, le rôle caché de Rabelais en tant qu'homme d'atelier, qui travaille en silence comme éditeur dans les grandes imprimeries de l'humanisme lyonnais. C'est la part la moins connue des activités de Rabelais, mais qui est, à l'heure actuelle, la plus étudiée ${ }^{46}$. Cette devise de l'éditeur est celle d'une troisième persona de Rabelais, qui a sa place à côté de ces deux autres masques, qui sont infiniment plus ostensibles : ceux de Rabelais et d'Alcofrybas.

\footnotetext{
${ }^{45}$ Menini, « Rabelais, homme d'atelier(s) », art. cit., p. 458.

${ }^{46} \mathrm{Je}$ signale la récente journée d'étude: TYХH АГА $\Theta \mathrm{H} \Xi \mathrm{YN} \Theta \mathrm{E} \Omega$. Rabelais éditeur des Anciens et des Modernes (Sorbonne, 17 avril 2015: http://officinedemercure.org/wpcontent/uploads/2015/03/Programme.pdf).
} 